\title{
Effect of Betatron Motion on the Septum Flux in Superslow Extraction at the SSC*
}

\author{
Barry S. Newberger \\ Institute for Fusion Studies and Department of Physics \\ University of Texas-Austin, Austin, TX 78712 USA \\ H.-J. Shih \\ Superconducting Super Collider Laboratory ${ }^{\dagger}$ \\ 2550 Beckleymeade Ave., Dallas, TX 75237 USA \\ J. A. Ellison \\ Department of Mathematics and Statistics \\ University of New Mexico, Albuquerque, NM 87131 USA
}

\begin{abstract}
A scheme for the superslow extraction of protons from the SSC rings for high energy test beam or precision fixed-target B physics is currently being investigated. ${ }^{1}$ The flux onto the extraction septum (a curved Si crystal) is to be generated by diffusion produced by noise injected into the RF system. The extraction rate depends on both the diffusion of the closed orbit (due to dispersion at the crystal) and the horizontal betatron amplitude of the diffusing protons. The diffusion of the closed orbit has previously been described by the onedimension longitudinal diffusion theory of Dôme and Krinsky and Wang. In this paper, we extend this theory to include the effect of the betatron motion. Comparisons with Monte Carlo tracking simulations are made.
\end{abstract}

\section{INTRODUCTION}

It has long been understood that noise in the RF system of a hadron storage ring leads to a slow loss of particles from the circulating beam bunches. ${ }^{1}$ It has become of considerable interest to exploit this to create an artificial halo for the purpose of extracting a low intensity beam for precision fixed target experiments or test beams at future hadron colliders. 2,3 A theoretical treatment of the loss process by a diffusion of the longitudinal action have been used for some time ${ }^{4-6}$ and do give results which qualitatively describe the observations. These theories have been strictly one-dimensional , taking no account of the betatron motion. Losses are assumed to occur at the separatrix of the longitudinal motion. In actuality, losses occur at a physical aperture stop. This might correspond to an extraction septum in the case of interest to us or, more generally, at any momentum scraper. Indeed, our recent results ${ }^{6}$ on diffusion in the presence of filtered noise suggest a scheme for scraping momentum tails. We have conducted Monte Carlo tracking studies in the SSC lattice. ${ }^{2,6}$

The betatron function can be large and the loss rates change significantly when the effect of the betatron motion is

\footnotetext{
* Supported in part by the SSC Laboratory and based in part on work supported by the Texas National Research Laboratory Commission under Grant No. RGFY 9234.

$\dagger$ Operated by the Universities Research Association, Inc., for the US. Department of Energy under Contract No. DE-AC35-89ER40486.
}

included. Recently, we have obtained a theory of the diffusion process which includes the effect of the betatron motion. ${ }^{7}$ Agreement between the particle losses in the simulation and the theory is excellent. In this paper, we briefly describe the theory and present new results on the "marginal" density in longitudinal action. These are also compared with the Monte Carlo tracking results and again the results agree.

\section{DIFFUSION THEORY}

The theor $y^{4-6}$ of longitudinal dynamics in a noisy RF system leads to a description of the evolution of an ensemble by a diffusion in the action, $J$, which is a constant of the unperturbed motion. The time scales of the diffusion in action, the synchrotron period and the betatron oscillation period are disparate with $t_{d}>t_{S}>>t \beta$. Thus the collimation process sweeps a shell in the transverse phase space $(A, A+d A)$, where $A$ characterizes a Courant-Snyder invariant, and a shell $(J$, $J+d J)$ in the longitudinal phase space in a time $t, t_{S}<t<t_{d}$. The maximum betatron displacement is related to $A$ by $\left|x_{\beta}\right|_{\max }=\sqrt{\beta} A$ where $\beta$ is the usual betatron function.

In the presence of an aperture stop, a particle slowly diffuses toward the periphery of the beam pipe until it strikes the stop. We assume the "image" of the stop in momentum, $x_{c} / D$, is inside the bucket. Here $D$ is the dispersion at the position of the stop. The time it takes until the particle is lost, or conversely, the loss rate, depends on both the closed orbit position (equivalently, action) and the betatron displacement.

If we had the joint probability density of $J$ and $x_{\beta}$ we would have all the information needed. (Here and throughout the remainder of the paper for ease of notation we have written $x_{\beta}$ when we really mean $\left|x_{\beta}\right|_{\max }$.) By definition, the joint density can be obtained from the probability conditioned on $x_{\beta}, p\left(J \mid x_{\beta}\right)$ :

$$
p\left(J, x_{\beta}\right)=p\left(J \mid x_{\beta}\right) p\left(x_{\beta}\right) .
$$

where $p\left(x_{\beta}\right)$ is the marginal density of $x_{\beta}$. These must be probability densities and must be defined throughout the domain of definition of the pair of random variables $J, x_{\beta}$ which is the quadrant $J \geq 0, x_{\beta} \geq 0$.

Consider particles which, at a given time, $t$, have not yet reached the stop. These lie in a domain in the space of the random variables bounded by the coordinate axes and a curve $J_{b}\left(x_{\beta}\right)$ which is the action for a particle on an energy 
surface $k$ defined by $k_{b}\left(x_{\beta}\right) \equiv\left(x_{c}-x_{\beta}\right) / D \delta p_{s}$ where $k \equiv \delta p / \delta p_{s}, \delta p$ the momentum deviation and $\delta p_{s}$ the bucket half-height. For these particles, the conditional density satisfies a diffusion equation of the form considered in earlier work ${ }^{4-6}$ :

$$
\frac{\partial}{\partial t} p\left(J, t \mid x_{\beta}\right)=\frac{\partial}{\partial J}\left[D(J) \frac{\partial}{\partial J} p\left(J, t \mid x_{\beta}\right)\right]
$$

but with boundary condition:

$$
p\left(J_{b}, t x_{\beta}\right)=0 .
$$

For these particles, the marginal density in $x_{\beta}$ is just the initial distribution.

In the remainder of the domain of the random variables, the conditional density is a $\delta$-function. It is fixed by the requirement that $p\left(x_{\beta}\right)$ be a marginal density; that is, an integral over all $J>0$ of the joint density.

In general, the diffusion equation must be solved numerically. However, analytic solutions have been obtained for diffusion coefficient linear in the action and quadratic in the action. In the former, straightforward separation of variables gives a solution ${ }^{4}$ as a Fourier-Bessel series. In the latter, a Fourier integral representation exists. ${ }^{8}$ While neither of these exactly correspond to simple noise spectral densities, the diffusion coefficient for small action is linear in the case of white phase noise and quadratic for white amplitude noise. ${ }^{6}$ Furthermore, we find the diffusion coefficient for white phase and for white amplitude noise reasonably can be fit over most of the bucket by linear and quadratic approximations respectively; the fit is very good in the latter case. This is illustrated in Fig. 1 where we have shown the diffusion coefficient in the cases of white phase and amplitude noise along with their approximations. The action is normalized to its value at the boundary $J_{b}$ for a zero betatron amplitude particle. We will now present results obtained from these solutions by use of Eq. (1) and which can be compared to data we obtain from our Monte Carlo simulations.

\section{THEORETICAL RESULTS}

There are several quantities which typically are of interest when we consider the results of a Monte Carlo extraction simulation. 2,6 of these, the number of particles reaching the septum at a given time and the distribution in action of the circulating particles are calculable from the diffusion theory. (Other data, such as the initial phase-space coordinates of the particles striking the septum and the distribution of hits on the septum are outside of the scope of a diffusion theory.) In this section, we compare the results of the diffusion theory with the Monte Carlo data.

The number of particles which have hit the septum up to a time, $t$, is found by integrating the joint density, Eq. (1) over the region of the random variable space, $S$, occupied by the circulating particles and applying conservation of probability:

$$
N(t)=1 .-\iint_{s} p\left(J, t \mid x_{\beta}\right) p\left(x_{\beta}\right) d J d x_{\beta} .
$$

Explicit expressions have been given in Ref. 7. Here we summarize the results ${ }^{7}$ for both white phase and white amplitude noise in Table 1. A typical Monte Carlo simulation follows 1000 tracks for about a million turns or about 5 minutes in real time. Obviously, the simulation extraction rates are substantially higher than would be employed in actual operation but computational constraints necessitate this. This underscores the importance of a theory which allows us to predict the behavior of the extraction process with realistic operating parameters.

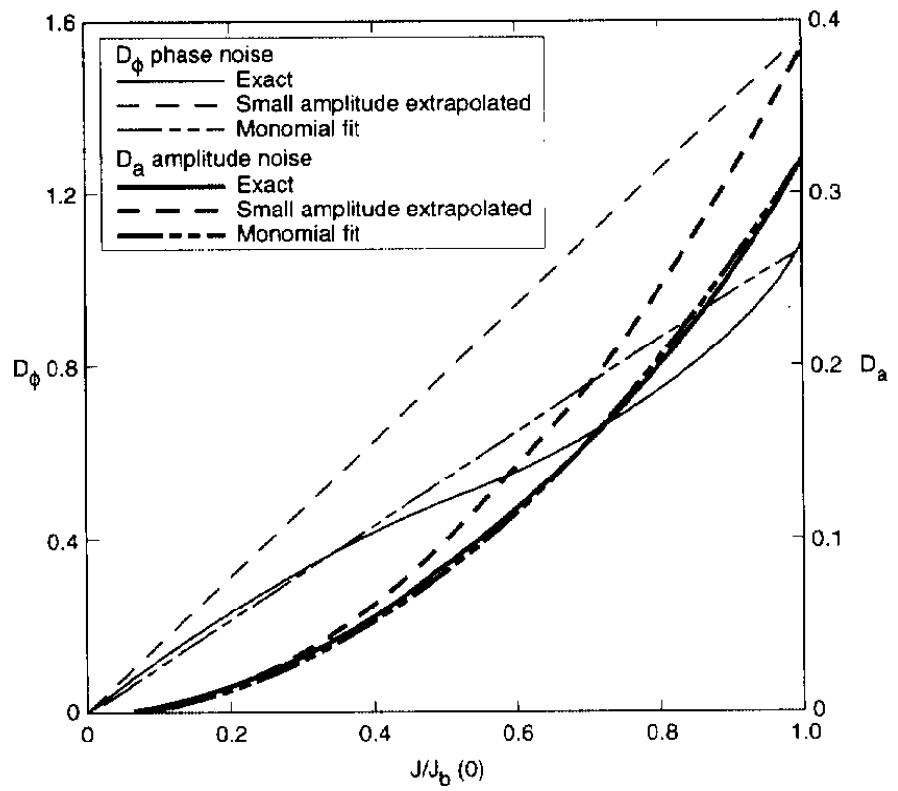

Figure 1. Diffusion coefficients for white noise.

Referring to the table, the first figure in the column of theoretical values is the result using the extrapolated small amplitude diffusion coefficient and the second value was obtained by using the diffusion coefficient fit by forcing the value of the monomial (linear or quadratic as appropriate) approximation to agree with the actual value at $J_{b}(0)$. The simulation results are from a single realization of the random process. The machine parameters are the nominal values for the SSC collider rings, $\sigma_{\delta p}=5 \times 10^{-5}, \varepsilon_{X}=1 \pi \mathrm{mm}$-mrad. The value of $\beta_{x}$ at the aperture stop was $1385 \mathrm{~m}$ in all cases but one. The exceptional case is indicated. The dispersion $D=4 \mathrm{~m}$. For both simulation and theory, thirty-one transient particles have been taken into account. These are particles which are initialized outside the septum and consequently are lost in a few turns independent of the noise. The agreement between the simulation and theory is generally very good particularly for the monomial fits.

The distribution in action of circulating particles is given by:

$$
p(J, t)=\int_{0}^{x_{\beta}(J)} p\left(J, x_{\beta}^{\prime}, t\right) d x_{\beta}^{\prime},
$$

where $x_{\beta}(J)$ is defined by 


$$
J_{b}\left(x_{\beta}\right)=J
$$

We simply refer to this as the "marginal" distribution in action but, technically, this is incorrect. It is actually the joint probability of having the "action in an interval $d J$ about $J$ " and the particle being "inside the aperture".

Table 1

Values of $N$. Theory values as described in text.

\begin{tabular}{lclcrl}
\hline & \multicolumn{2}{c}{ Phase Noise } & \multicolumn{3}{c}{ Amplitude Noise } \\
$\sigma_{\phi}$ & $\mathrm{N}$ (sim) & $\mathrm{N}$ (theory) & $\sigma_{a}$ & $\mathrm{~N}(\mathrm{sim})$ & $\mathrm{N}$ (theory) \\
\hline \hline 0.2 & 584 & $732 / 595$ & 0.5 & 745 & $772 / 718$ \\
$0.2^{*}$ & 343 & $542 / 363$ & 0.2 & 182 & $211 / 178$ \\
0.1 & 187 & $261 / 179$ & 0.1 & 67 & $56 / 48$ \\
0.05 & 51 & $69 / 49$ & 0.05 & 22 & $19 / 17$ \\
0.02 & 13 & $18 / 14$ & 0.02 & 7 & $7 / 6$ \\
\hline
\end{tabular}

${ }^{*} \beta_{x}=346 \mathrm{~m}$

We have calculated $p(J, t)$ for both phase and amplitude noise. The integral is done numerically using a standard Romberg routine on a personal computer. The results are shown in Fig. 2a and Fig. $2 \mathrm{~b}$ for white phase noise and white amplitude noise respectively. The solid curves are for the diffusion coefficient given by the fit while the result using small amplitude extrapolated diffusion coefficient is the dashed curve. These are superimposed on the histogram from the Monte Carlo tracking. The phase noise corresponds to a an rms phase $\sigma_{\phi}=0.1 \mathrm{rad}$. The rms relative amplitude noise modulation $\sigma_{\mathrm{a}}=0.2$. In the Monte Carlo results, bar heights of approximately $1 . \times 10^{-3}$ correspond to single particles. The agreement is seen to be quite good. Insofar as the marginal densities are concerned, the differences between the result using the small amplitude extrapolation or the fit for the diffusion coefficient is small.

\section{CONCLUSION}

We have described a theory of the noise induced diffusion in the longitudinal phase space which accounts for the effect of the betatron motion. The two degrees-of-freedom are coupled at a position with dispersion by any real stop. The problem of interest contemplates noise deliberately introduced into the RF system for superslow extraction of low intensity proton beams. The agreement with Monte Carlo tracking simulations is excellent. The theory could also be applied to momentum scrapers, when the momentum halo production can be described by a diffusive process. The formalism should also apply when the marginal density in $x_{\beta}$ has time dependence provided the evolution is independent of $J$.

\section{REFERENCES}

[1] D. Boussard, G. Dôme and C. Graziani, "The Influence of RF Noise on the Lifetime of Bunched Proton Beams," in 11th International Conference on High-Energy Accelerators, edited by W. S. Newman, (Birkhäuser, Basel, 1980), p. 620.
[2] B.S. Newberger, H.-J. Shih and J.A. Ellison, Nucl. Instrum. Methods A325, 9 (1993).

[3] S. Weisz, et. al., "Proton Extraction from the CERN-SPS by a Bent Crystal" this conference.

[4] G. Dôme, "Theory of RF Acceleration and RF Noise," in Accelerator School for Antiprotons for Colliding Beam Facilities, CERN Report 84-15, 1984, p. 215.

[5] S. Krinsky and J. M. Wang, Particle Accelerators 12, 107 (1982).

[6] H.-J. Shih, J. Ellison, B. Newberger and R. Cogburn, "Longitudinal Beam Dynamics with RF Noise," SSC Laboratory Report SSCL-578, July 1992, submitted to Particle Accelerators.

[7] B. Newberger, J. Ellison and H.-J. Shih, "Effect of Betatron Motion on Particle Loss Due to Longitudinal Diffusion in High-energy Colliders," SSC Laboratory Report SSCL-preprint-205, March 1993, submitted to Physical Review Letters.

[8] H.-J. Shih, J. Ellison and W. Schiesser, "Reliability of Numerical Solutions of a Diffusion Equation Modeling RF Noise-Induced Dilution in Particle Beams" in Advances in Computer Methods for Partial Differential Equations VII, R. Vichnevetsky, D. Knight and G. Richter, eds. (IMACS, New Brunswick, 1992) p. 663.
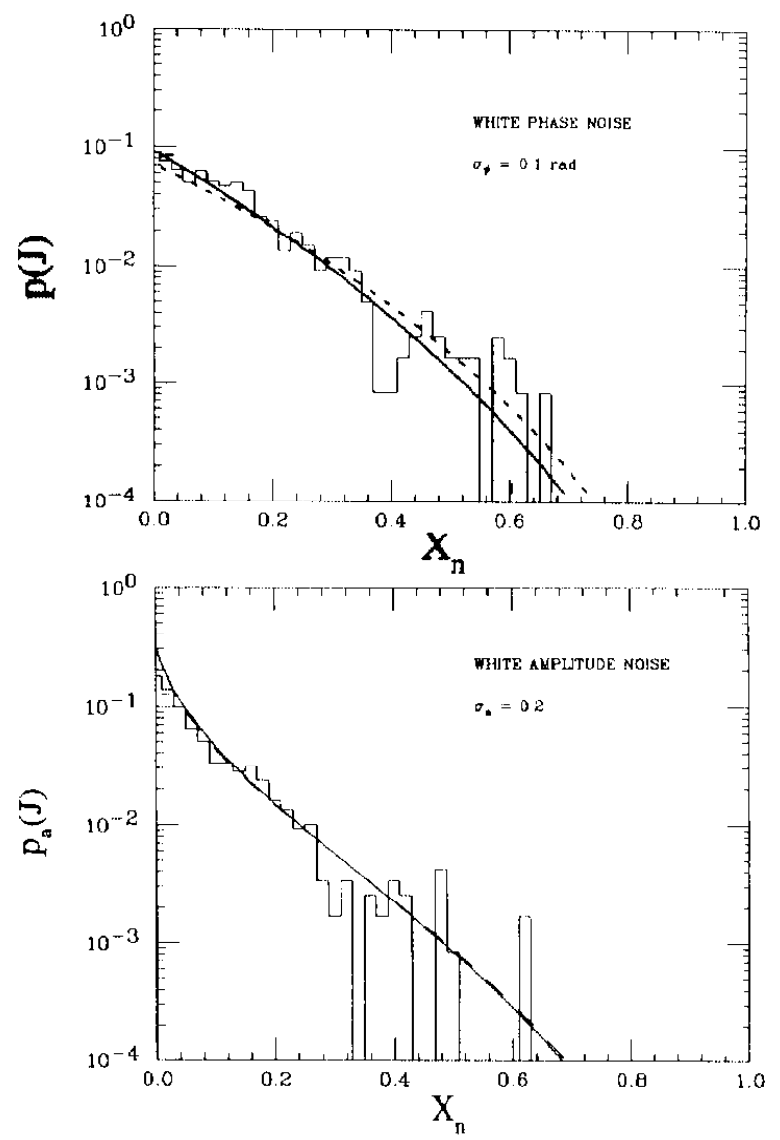

Figure 2. Marginal action density distributions: a) phase noise, $\sigma_{\phi}=0.1 \mathrm{rad}$ b) amplitude noise, $\sigma_{a}=0.2$. 